\title{
A SAT ENCODING FOR SOLVING GAMES WITH ENERGY OBJECTIVES
}

\author{
Raffaella Gentilini \\ Dip. di Matematica e Informatica, Universit'a di Perugia, \\ Via Vanvitelli 1, Perugia (IT)
}

\begin{abstract}
Recently, a reduction from the problem of solving parity games to the satisfiability problem in propositional logic (SAT) have been proposed in [5], motivated by the success of SAT solvers in symbolic verification. With analogous motivations, we show how to exploit the notion of energy progress measure to devise a reduction from the problem of energy games to the satisfiability problem for formulas of propositional logic in conjunctive normal form.
\end{abstract}

\section{INTRODUCTION}

Energy games (EG) are two-players games played on weighted graphs, where the integer weight associated to each edge represents the corresponding energy gain/loss. The arenas of energy games are endowed of two types of vertices: in player 0 (resp. player 1) vertices, player 0 (resp. player 1) chooses the successor vertex from the set of outgoing edges and the game results in an infinite path through the graph. Given an initial credit of energy c, the objective of player 0 is to maintain the sum of the weights (the energy level) positive. The decision problem for EG asks, given a weighted game graph with initial vertex $v_{0}$, if there exists an initial credit for which player 0 wins from $\nu_{0}$.

Energy games have been introduced in $[3,2]$ to model the synthesis problem within the design of reactive systems that work in resource-constrained environments. Beside their applicability to the modeling of quantitative problems for computer aided design, EG have tight connections with important problems in game theory and logic. For instance, they are log-space equivalent to mean-payoff games (MPG) [2], another kind of quantitative two-player game very well studied both in economics and in computer science. The latter are characterized by a theoretically engaging complexity status, being one of the few inhabitants of the complexity class NPीcoNP (for which the inclusion in $\mathrm{P}$ is still an open problem). Moreover, parity games $[4,6]-$ notoriously known as poly-time equivalent to the model-checking problem for the modal mucalculus - are in turn poly-time reducible to MPG and EG. It is a long-standing open question to know whether the model-checking problem for the modal mu-calculus is in P.

The algorithm with the currently best (pseudopolynomial) complexity for solving EG (and MPG via log-space reduction) is based on the so-called notion of energy progress measure [7].

David C. Wyld et al. (Eds) : CST, ITCS, JSE, SIP, ARIA, DMS - 2014

pp. 45-52, 2014. (C) CS \& IT-CSCP 2014

DOI : $10.5121 /$ csit.2014.4105 
Progress measures for weighted graphs are functions that impose local conditions to ensure global properties of the graph. A notion of parity progress measure [6] was previously exploited in [6] for the algorithmic analysis of parity games and reconsidered in [5] to devise a SAT encoding of the corresponding games, motivated by the considerable success that using SAT solvers has had in symbolic verification. As a matter of fact, clever heuristics implemented in nowadays SAT solvers can result in algorithms that are very efficient in practice. Furthermore, there are fragments of SAT that can be solved in polynomial time. Hence, the reduction in [5] opens up a new possibility for showing inclusion of parity games in $\mathrm{P}$.

Motivated by analogous reasons, in this paper we show how to exploit the notion of energy progress measure to devise a reduction from the problem of energy games to the satisfiability problem for formulas of propositional logic in conjunctive normal form. Tight upper bounds on the sizes of our reductions are also reported.

The paper is organized as follows.We recall the notions of energy games and energy progress measure in Section 2. Section 3 and Section 4 develop the reductions from energy games to difference logic and pure SAT, respectively, reporting tight bounds on the sizes of the corresponding reductions.

\section{PRELIMINARIES}

Game graphs A game graph is a tuple $\Gamma=\left(V, E, v_{0}, w,\left\langle V_{0}, V_{1}\right\rangle\right)$ where $G^{\Gamma}=$ $\left(V, E, v_{0}, w\right)$ is a weighted graph with weight function $w: E \rightarrow \mathbb{Z}$ and $\left\langle V_{0}, V_{1}\right\rangle$ is a partition of $V$ into the set $V_{0}$ of player- 0 vertices and the set $V_{1}$ of player- 1 vertices. An infinite game on $\Gamma$ is played for infinitely many rounds by two players moving a pebble along the edges of the weighted graph $G^{\Gamma}$. In the first round, the pebble is on some vertex $v \in V$. In each round, if the pebble is on a vertex $v \in V_{i}(i=0,1)$, then player $i$ chooses an edge $\left(v, v^{\prime}\right) \in E$ and the next round starts with the pebble on $v^{\prime}$. A play in the game graph $\Gamma$ is an infinite sequence $p=v_{0} v_{1} \ldots v_{n} \ldots$ such that $\left(v_{i}, v_{i+1}\right) \in E$ for all $i \geq 0$. A strategy for player $i(i=0,1)$ is a function $\sigma: V^{*} \cdot V_{i} \rightarrow V$, such that for all finite paths $v_{0} v_{1} \ldots v_{n}$ with $v_{n} \in V_{i}$, we have $\left(v_{n}, \sigma\left(v_{0} v_{1} \ldots v_{n}\right)\right) \in E$. We denote by $\Sigma_{i}(i=0,1)$ the set of strategies for player $i$. A strategy $\sigma$ for player $i$ is memoryless if $\sigma(p)=\sigma\left(p^{\prime}\right)$ for all sequences $p=v_{0} v_{1} \ldots v_{n}$ and $p^{\prime}=v_{0}^{\prime} v_{1}^{\prime} \ldots v_{m}^{\prime}$ such that $v_{n}=v_{m}^{\prime}$. We denote by $\Sigma_{i}^{M}$ the set of memoryless strategies of player $i$. A play $v_{0} v_{1} \ldots v_{n} \ldots$ is consistent with a strategy $\sigma$ for player $i$ if $v_{j+1}=\sigma\left(v_{0} v_{1} \ldots v_{j}\right)$ for all positions $j \geq 0$ such that $v_{j} \in V_{i}$. Given an initial vertex $v \in V$, the outcome of two strategies $\sigma_{1} \in \Sigma_{1}$ and $\sigma_{2} \in \Sigma_{2}$ in $v$ is the (unique) play outcome ${ }^{\Gamma}\left(v, \sigma_{0}, \sigma_{1}\right)$ that starts in $v$ and is consistent with both $\sigma_{0}$ and $\sigma_{1}$. Given a memoryless strategy $\pi_{i}$ for player $i$ in the game $\Gamma$, we denote by $G^{\Gamma}\left(\pi_{i}\right)=\left(V, E_{\pi_{i}}, w\right)$ the weighted graph obtained by removing from $G^{\Gamma}$ all edges $\left(v, v^{\prime}\right)$ such that $v \in V_{i}$ and $v^{\prime} \neq \pi_{i}(v)$.

Energy Games [3,2] An energy game (EG) is an infinite game on the game graph $\Gamma$, where the goal of player 0 is to construct an infinite play $v_{0} v_{1} \ldots v_{n} \ldots$ such that for some initial credit $c \in \mathbb{N}$ : 


$$
c+\sum_{i=0}^{j} w\left(v_{i}, v_{i+1}\right) \geq 0 \text { for all } j \geq 0
$$

The quantity $c+\sum_{i=0}^{j-1} w\left(v_{i}, v_{i+1}\right)$ is called the energy level of the play prefix $v_{0} v_{1} \ldots v_{j}$. Given a credit $c$, a play $p=v_{0} v_{1} \ldots$ is winning for player 0 if it satisfies (1), otherwise it is winning for player 1 . A vertex $v \in V$ is winning for player $i$ if there exists an initial credit $c$ and a winning strategy for player $i$ from $v$ for credit $c$. In the sequel, we denote by $W_{i}$ the set of winning states for player $i$. Energy games are memoryless determined [2], i.e. for all $v \in V$, either $v$ is winning for player 0 , or $v$ is winning for player 1 , and memoryless strategies are sufficient.

Theorem 1 ([2]). Let $\Gamma=\left(V, E, v_{0}, w,\left\langle V_{0}, V_{1}\right\rangle\right)$ be an $\mathrm{EG}$, for all $v \in V$, the following four statements are equivalent:

- $\exists \sigma_{0} \in \Sigma_{0} \cdot \forall \sigma_{1} \in \Sigma_{1} \cdot$ outcome $^{\Gamma}\left(v, \sigma_{0}, \sigma_{1}\right)$ is winning for player $O$;

- $\forall \sigma_{1} \in \Sigma_{1} \cdot \exists \sigma_{0} \in \Sigma_{0} \cdot$ outcome $^{\Gamma}\left(v, \sigma_{0}, \sigma_{1}\right)$ is winning for player $O$;

- $\exists \pi_{0} \in \Sigma_{0}^{M} \cdot \forall \pi_{1} \in \Sigma_{1}^{M} \cdot$ outcome $^{\Gamma}\left(v, \pi_{0}, \pi_{1}\right)$ is winning for player $O$;

- $\forall \pi_{1} \in \Sigma_{1}^{M} \cdot \exists \pi_{0} \in \Sigma_{0}^{M} \cdot$ outcome $^{\Gamma}\left(v, \pi_{0}, \pi_{1}\right)$ is winning for player 0 ;

Using the memoryless determinacy of energy games, the authors of [7] derived the next characterizaion lemma for EG winning strategies.

Lemma 1 ([7]). Let $\Gamma=\left(V, E, w,\left\langle V_{0}, V_{1}\right\rangle\right)$ be an $\mathrm{EG}$. For all vertices $v \in V$, for all memoryless strategies $\pi_{0} \in \Sigma_{0}^{M}$ for player 0 , the strategy $\pi_{0}$ is winning from $v$ if and only if all cycles reachable from $v$ in the weighted graph $G^{\Gamma}\left(\pi_{0}\right)$ are nonnegative.

Given the energy game $\Gamma=\left(V, E, v_{0}, w,\left\langle V_{0}, V_{1}\right\rangle\right)$, the EG decision problem asks wether $v_{0}$ is winning for player 0 . Such a problem is polynomially equivalent to the corresponding decision problem for so-called meanpayoff games $[2,1]$.

The algorithm with the currently best (pseudopolynomial) complexity for solving energy games is based on the so-called notion of small energy progress measure [7]. Intuitively, the latter is a condition locally defined on the vertices of the given game graph, tailored to witness the global absence of negative cycles within the subgame induced by a proper strategy for player 0 (cfr. the characterization lemma 1). Formally, the notion of small progress measure is recalled in Definition 1 (below) and relies on the following notation. Given $\Gamma=\left(V, E, v_{0}, w,\left\langle V_{0}, V_{1}\right\rangle\right)$, denote by $\mathcal{C}_{\Gamma}$ the following set:

$$
\mathcal{C}_{\Gamma}=\left\{n \in \mathbb{N} \mid n \leq \mathcal{M}_{G^{\Gamma}}\right\} \cup\{\top\}
$$

where:

$$
\mathcal{M}_{G_{I}}=\sum_{v \in V} \max \left(\{0\} \cup\left\{-w\left(v, v^{\prime}\right) \mid\left(v, v^{\prime}\right) \in E\right\}\right)
$$

Moreover, denote by $\preceq$ the total order on $\mathcal{C}_{\Gamma}$ defined by $x \preceq y$ if and only if either $y=\top$ or $x \leq y \leq \mathcal{M}_{G^{\Gamma}}$. Finally, let $\ominus: \mathcal{C}_{\Gamma} \times \mathbb{Z} \rightarrow \mathcal{C}_{\Gamma}$ be the operator such that for all $a \in \mathcal{C}_{\Gamma}$ and $b \in \mathbb{Z}$ : 


$$
a \ominus b= \begin{cases}\max (0, a-b) & \text { if } a \neq \top \text { and } a-b \leq \mathcal{M}_{G^{T}} \\ \top & \text { otherwise }\end{cases}
$$

Definition 1 ([7]). Let $\Gamma=\left(V, E, v_{0}, w,\left\langle V_{0}, V_{1}\right\rangle\right)$ be an EG. A function $f: V \rightarrow \mathcal{C}_{\Gamma}$ is a small energy progress measure for $\Gamma$ if and only if the following conditions hold:

- if $v \in V_{0}$, then $f(v) \succeq f\left(v^{\prime}\right) \ominus w\left(v, v^{\prime}\right)$ for some $\left(v, v^{\prime}\right) \in E$;

- if $v \in V_{1}$, then $f(v) \succeq f\left(v^{\prime}\right) \ominus w\left(v, v^{\prime}\right)$ for all $\left(v, v^{\prime}\right) \in E$.

Given a small energy progress measure $f$ for the game graph $\Gamma=\left(V, E, v_{0}, w,\left\langle V_{0}, V_{1}\right\rangle\right)$, we denote by $V_{f}$ the set of states $V_{f}=\{v \mid f(v) \neq \top\}$. A memoryless strategy $\pi_{0}^{f}: V_{0} \rightarrow V$ for player 0 is called compatible with $f$ whenever for all $v \in V_{0}$, if $\pi_{0}^{f}(v)=v^{\prime}$ then $f(v) \succeq f\left(v^{\prime}\right) \ominus w\left(v, v^{\prime}\right)$. The following property holds [7]: if $\pi_{0}^{f}$ is a strategy for player 0 compatible with the energy progress measure $f$, then $\pi_{0}^{f}$ is a winning strategy for player 0 from all vertices in $V_{f}$. Formally:

Theorem 2 ([7]). Let $\Gamma=\left(V, E, v_{0}, w,\left\langle V_{0}, V_{1}\right\rangle\right)$ be an EG. For all small energy progress measures $f$ for $\Gamma$, if $\pi_{0}^{f}$ is a strategy for player 0 compatible with $f$, then $\pi_{0}^{f}$ is a winning strategy for player 0 from all vertices $v \in V_{f}$, i.e. $V_{f} \subseteq W_{0}$. Moreover, $\Gamma$ admits a small energy progress measure $f$ such that $V_{f}=W_{0}$.

\subsection{Difference Logic}

Let $\mathcal{B}=\left\{b_{1}, \ldots, b_{n}\right\}$ be a set of boolean variables and $\mathcal{X}=\left\{x_{1}, \ldots, x_{n}\right\}$ be a set of integer variables. The set of atomic formulas of difference logic consists of the boolean variables in $\mathcal{B}$ and integer constraints of the form $x_{i}-x_{j} \geq c, c \in \mathbb{Z}$.

The set $\mathcal{F}$ of difference logic formulas is the smallest set containing the atomic formulas which is closed under negation and conjunction ( the boolean connectives $\vee, \rightarrow, \leftrightarrow$ are defined in the usual way in terms of the operators of negation and conjunction $\wedge, \neg)$. A $(\mathcal{B}, \mathcal{X})$ valuation consists of two functions (overloaded with the name $\alpha), \alpha: \mathcal{B} \rightarrow\{1,0\}, \alpha: \mathcal{X} \rightarrow \mathcal{Z}$. The valuation is extended to all difference logic formulas by letting $\alpha\left(x_{i}-x_{j} \geq c\right)=1$ if and only if $\alpha\left(x_{i}\right)-\alpha\left(x_{j}\right) \geq c$ and applying the obvious rules for boolean connectives. A difference logic formula $\phi$ is satisfied by a valuation $\alpha$ if and only if $\alpha(\phi)=1$. A formula $\phi$ is satisfiable if it admits a satisfying valuation. The satisfyiability problem for difference logic is NP-complete [8].

\section{ENCODING EG WINNING STRATEGIES IN DIFFERENCE LOGIC}

In this section we show how to derive a difference logic formula $\phi_{\Gamma}$ from a given energy game $\Gamma=\left(V, E, v_{0}, w,\left\langle V_{0}, V_{1}\right\rangle\right)$ such that $\phi_{\Gamma}$ is satisfiable if and only if player 0 has a winning strategy on $\Gamma$.

In particular, the difference logic formula $\phi_{\Gamma}$ uses the set of $|E|$ integer constants $\left\{w_{(v, z)} \mid(v, z) \in E\right\}$ and ranges over the following set of boolean and integer variables: 
- for each $v \in V$, there is a boolean variable $n_{v}$ and an integer variable $c_{v}$

- for aech edge $(v, z) \in E$, there is a boolean variable $m_{(v, z)}$

Given the above variables, $\phi_{\Gamma} \equiv n_{v_{0}} \wedge \phi_{0} \wedge \phi_{1} \wedge \phi_{\sigma} \wedge \phi_{e}$ is the conjunction of five subformulas, where $\phi_{0}, \phi_{1}, \phi_{\sigma}, \phi_{e}$ are defined as follows:

- $\phi_{0} \equiv \bigwedge_{v \in V_{0}}\left(n_{v} \rightarrow \bigvee_{(v, z) \in E} m_{(v, z)}\right)$

- $\phi_{1} \equiv \bigwedge_{v \in V_{0}}\left(n_{v} \rightarrow \bigwedge_{(v, z) \in E} m_{(v, z)}\right)$

- $\phi_{\rho} \equiv \bigvee_{\substack{v \in V \\ v \neq v_{0}}}\left(\left(\bigvee_{(v, z) \in E} m_{(v, z)}\right) \rightarrow n_{z}\right)$

- $\phi_{e} \equiv \underset{(v, z) \in E}{\bigvee}\left(m_{(v, z)} \rightarrow \psi_{(v, z)}\right)$

- $\psi_{(v, z)} \equiv c_{v}+w_{(v, z)} \geq c_{z}$

Theorem 3. Player 0 has a winning strategy in the energy game $\Gamma=\left(V, E, v_{0}, w,\left\langle V_{0}, V_{1}\right\rangle\right)$ if and only if the difference logic formula $\phi_{\Gamma}$ is satisfiable.

Proof. $(\Rightarrow)$ Let $G_{\Gamma}(\pi)$ be the graph induced by a winning strategy $\pi$ for player 0 on the energy game $\Gamma=\left(V, E, v_{0}, w,\left\langle V_{0}, V_{1}\right\rangle\right)$. Consider the assignment $\alpha$ to the variables of $\phi_{\Gamma}$ defined as follows: for each boolean variable $n_{v}$ (resp. $\left.m_{(v, z)}\right)$ let $\alpha\left(n_{v}\right)=1$ (resp. $\alpha\left(m_{(v, z)}\right)=1$ ) if and only if $v$ is a node (resp. $(v, z)$ is an edge) of $G_{\Gamma}(\pi)$. By definition of $G_{\Gamma}(\pi)$, the assignment $\alpha$ satisfies $n_{v_{0}} \wedge \phi_{0} \wedge \phi_{1}$. By Theorem $2, G_{\Gamma}(\pi)$ admits a small progress measure function $f: W \rightarrow \mathcal{M}_{G_{T}(\pi)}$, where $W$ is the set of vertices of $G_{\Gamma}(\pi)$. For each integer variable $c_{v}$ in $\phi_{\Gamma}$, define $\alpha\left(c_{v}\right)=f(v)$ if $v \in W$. Since $\pi$ is a winning strategy on $\Gamma$ for player 0 , the assignment $\alpha$ satisfies also the last conjunct $\phi_{\sigma}$ in $\phi_{\Gamma}$. Therefore, $\alpha \vDash \phi_{\Gamma}$.

$(\Leftarrow)$ Suppose that $\alpha$ is a satisfying variable assignment of $\phi_{\Gamma}$. Define the following game $\Gamma^{\prime}=\left(V^{\prime}, E^{\prime}, v_{0}, w^{\prime},\left\langle V_{0}^{\prime}, V_{1}^{\prime}\right\rangle\right): v \in V^{\prime}$ (resp. $\left.(v, z) \in E^{\prime}\right)$ if and only if $\alpha\left(n_{v}\right)=$ 1 (resp. $\alpha\left(m_{(v, z)}=1\right)$ ) and for each $(v, z) \in E^{\prime}$ let $w^{\prime}(v, z)=w_{(v, z)}$. Since $\alpha$ satisfies $v_{v_{0}} \wedge \phi_{0} \wedge \phi_{1} \wedge \phi_{\sigma}$, we derive that $\Gamma^{\prime}$ is a non empty subgame of $\Gamma$. Hence, since $\alpha$ satisfies also $\phi_{e}$, by Theorem 2 we deduce that $V^{\prime} \subseteq W_{0}$ and $\Gamma^{\prime}$ induces a winning strategy for player 0 on $\Gamma$.

Theorem 4. Given an energy game $\Gamma=\left(V, E, v_{0}, w,\left\langle V_{0}, V_{1}\right\rangle\right)$, the size of the difference logic formula $\phi_{\Gamma}$ is $\mathcal{O}(|E|)$, even if $\phi_{\Gamma}$ is required to be in CNF.

Proof. Each subformula $\phi_{0} \wedge \phi_{1}, \phi_{\sigma}, \phi_{e}$ has size $\mathcal{O}(|E|)$, while the remaining conjunct $n_{v_{0}}$ in $\phi_{\Gamma}$ has size 1. $\phi_{\Gamma}$ can be rewritten in CNF with a constant blow up by reformulating the conjuncts $\phi_{0}, \phi_{1}, \phi_{\sigma}$ and $\phi_{e}$ using the boolean equivalences:

$$
\begin{aligned}
& \chi \rightarrow(\phi \wedge \psi) \equiv(\chi \rightarrow \phi) \wedge(\chi \rightarrow \psi) \\
& (\phi \vee \psi) \rightarrow \chi) \equiv(\phi \rightarrow \chi) \wedge(\psi \rightarrow \chi)
\end{aligned}
$$




\section{SOLVING ENERGY GAMES BY A REDUCTION TO SAT}

In this section, we present an encoding for the difference logic formula $\phi_{\Gamma}$ associated to a given energy game $\Gamma$ into propositional logic, i.e. the subset of difference logic with boolean variables only. Clearly, all that remains to be done is to translate the integer variables and the constraints on them of the form $c_{v}+w_{(v, z)} \geq c_{z}$ inside the conjuct $\phi_{e}$ in $\phi_{\Gamma}$.

Let $\Gamma=\left(V, E, v_{0}, w,\left\langle V_{0}, V_{1}\right\rangle\right)$ be the energy game underlying $\phi_{\Gamma}$. By Theorem 2 the domain of the integer variables in $\phi_{\Gamma}$ can be bounded by $\mathcal{M}_{G_{T}} \leq V \cdot W$, where $W$ is the maximum absolute weight in $\Gamma$. Let $k=\left\lceil\log \left(\mathcal{M}_{G_{\Gamma}}+W\right)\right\rceil$ be the number of bits necessary to code $\mathcal{M}_{G_{T}}, W$.

For each edge $(v, z) \in E$, let $\bar{w}_{(v, z)}=w_{1} \ldots w_{k}$ be the boolean encoding of $\left|w_{(v, z)}\right|$ (using $k$ boolean variables), let $e_{1}^{v}, \ldots e_{k}^{v}, e_{1}^{z}, \ldots e_{k}^{z}, s_{1}^{(v, z)} \ldots s_{k}^{(v, z)}, r_{0}^{(v, z)} \ldots r_{k}^{(v, z)}$, be further boolean variables and consider the following propositional formulas:

- If $w_{(v, z)} \geq 0$ :

- $\operatorname{CURRY}(v, z, k) \equiv \neg r_{k}^{(v, z)}$

- for $i=k \ldots 1$ :

$$
\begin{gathered}
\operatorname{suM}(v, z, i) \equiv s_{i}^{(v, z)} \Leftrightarrow\left(\neg e_{i}^{v} \wedge \neg w_{i} \wedge \neg r_{i}^{(v, z)}\right) \vee\left(\neg e_{i}^{v} \wedge w_{i} \wedge \neg r_{i}^{(v, z)}\right) \\
\vee\left(e_{i}^{v} \wedge \neg w_{i} \wedge \neg r_{i}^{(v, z)}\right) \vee\left(e_{i}^{v} \wedge w_{i} \wedge r_{i}^{(v, z)}\right) \\
\operatorname{CURRY}(v, z, i-1) \equiv r_{i-1}^{(v, z)} \Leftrightarrow\left(\neg e_{i}^{v} \wedge w_{i} \wedge r_{i}^{(v, z)}\right) \vee\left(e_{i}^{v} \wedge \neg w_{i} \wedge r_{i}^{(v, z)}\right) \\
\vee\left(e_{i}^{v} \wedge w_{i} \wedge \neg r_{i}^{(v, z)}\right) \vee\left(e_{i}^{v} \wedge w_{i} \wedge r_{i}^{(v, z)}\right)
\end{gathered}
$$

- $\operatorname{CURRY}(v, z, 0) \equiv \neg r_{0}^{(v, z)}$

- $\operatorname{GEQ}(v, z, 1) \equiv s_{1}^{(v, z)} \Rightarrow e_{1}^{z}$

- for $i=k \ldots 1$ :

$$
\operatorname{GEQ}(v, z, i) \equiv\left(s_{i}^{(v, z)} \Rightarrow e_{i}^{z}\right) \wedge\left(\left(s_{i}^{(v, z)} \vee \neg e_{i}^{z}\right) \Rightarrow \operatorname{GEQ}(v, z, i-1)\right.
$$

- If $w_{(v, z)}<0$ :

- $\operatorname{CURRY}(v, z, k) \equiv \neg r_{k}^{(v, z)}$

- for $i=k \ldots 1$ :

$$
\begin{aligned}
& \operatorname{SUM}(v, z, i) \equiv s_{i}^{(v, z)} \Leftrightarrow\left(\neg e_{i}^{z} \wedge \neg w_{i} \wedge \neg r_{i}^{(v, z)}\right) \vee\left(\neg e_{i}^{z} \wedge w_{i} \wedge \neg r_{i}^{(v, z)}\right) \\
& \vee\left(e_{i}^{z} \wedge \neg w_{i} \wedge \neg r_{i}^{(v, z)}\right) \vee\left(e_{i}^{z} \wedge w_{i} \wedge r_{i}^{(v, z)}\right) \\
& \operatorname{CURRY}(v, z, i-1) \equiv r_{i-1}^{(v, z)} \Leftrightarrow\left(\neg e_{i}^{z} \wedge w_{i} \wedge r_{i}^{(v, z)}\right) \vee\left(e_{i}^{z} \wedge \neg w_{i} \wedge r_{i}^{(v, z)}\right) \\
& \vee\left(e_{i}^{z} \wedge w_{i} \wedge \neg r_{i}^{(v, z)}\right) \vee\left(e_{i}^{z} \wedge w_{i} \wedge r_{i}^{(v, z)}\right) \\
& \operatorname{GEQ}(v, z, i) \equiv\left(e_{i}^{v}\right) \Rightarrow s_{i}^{(v, z)} \wedge\left(\left(e_{i}^{v} \vee \neg s_{i}^{(v, z)}\right) \Rightarrow \operatorname{GEQ}(v, z, i-1)\right.
\end{aligned}
$$


Let $\phi_{\Gamma}^{\prime}$ be the propositional logic formula obtained by replacing each integer constraint in $\phi_{\Gamma}$ of the form $c_{v}+w_{(v, z)} \geq c_{z}$ by the propositional formula $\operatorname{GEQ}(v, z, k)$

Theorem 5. Player 0 has a winning strategy in the energy game $\Gamma=\left(V, E, v_{0}, w,\left\langle V_{0}, V_{1}\right\rangle\right)$ if and only if the propositional logic formula $\phi_{\Gamma}^{\prime}$ is satisfiable.

Proof. $(\Rightarrow)$ Let $G_{\Gamma}(\pi)$ be the graph induced by a winning strategy $\pi$ for player 0 on the energy game $\Gamma=\left(V, E, v_{0}, w,\left\langle V_{0}, V_{1}\right\rangle\right)$. Consider the assignment $\alpha$ to the variables of $\phi_{\Gamma}$ defined as follows: for each boolean variable $n_{v}$ (resp. $m_{(v, z)}$ ) let $\alpha\left(n_{v}\right)=1$ (resp. $\alpha\left(m_{(v, z)}\right)=1$ ) if and only if $v$ is a node (resp. $(v, z)$ is an edge) of $G_{\Gamma}(\pi)$. By Theorem 3, the assignment $\alpha$ satisfies $n_{v_{0}} \wedge \phi_{0} \wedge \phi_{1}$. By Theorem 2, $G_{\Gamma}(\pi)$ admits a small progress measure function $f: W \rightarrow \mathcal{M}_{G_{T}(\pi)}$, where $W$ is the set of vertices of $G_{\Gamma}(\pi)$. For each $(v, z) \in E$ such that $w(v, z) \geq 0$ (resp. $w(v, z)<0$ ) :

- let $\alpha\left(e_{1}^{v}\right), \ldots \alpha\left(e_{k}^{v}\right)$ be the boolean code of $f(v)$

- let $\alpha\left(e_{1}^{z}\right), \ldots \alpha\left(e_{k}^{z}\right)$ be the boolean code of $f(z)$

- let $\alpha\left(s_{1}^{(v, z)}\right), \ldots \alpha\left(s_{k}^{(v, z)}\right), \alpha\left(r_{0}^{(v, z)}\right), \ldots \alpha\left(r_{k}^{(v, z)}\right)$ be the boolean code of the sum $f(v)+w(v, z)$ (resp. $f(z)+(-w(v, z)))$ and the corresponding curry bits.

Since $\pi$ is a winning strategy on $\Gamma$ for player 0 , the assignment $\alpha$ satisfies the propositional formula $\operatorname{GEQ}(v, z, k)$. Therefore, $\alpha \vDash \phi_{\Gamma}$.

$(\Leftarrow)$ Suppose that $\alpha$ is a satisfying variable assignment of $\phi_{\Gamma}$. Define the following game $\Gamma^{\prime}=\left(V^{\prime}, E^{\prime}, v_{0}, w^{\prime},\left\langle V_{0}^{\prime}, V_{1}^{\prime}\right\rangle\right): v \in V^{\prime}$ (resp. $\left.(v, z) \in E^{\prime}\right)$ if and only if $\alpha\left(n_{v}\right)=$ 1 (resp. $\alpha\left(m_{(v, z)}=1\right)$ ) and for each $(v, z) \in E^{\prime}$ let $w^{\prime}(v, z)=w_{(v, z)}$. Since $\alpha$ satisfies $v_{v_{0}} \wedge \phi_{0} \wedge \phi_{1} \wedge \phi_{\sigma}$, we derive that $\Gamma^{\prime}$ is a non empty subgame of $\Gamma$. Hence, since $\alpha$ satisfies also $\phi_{e}$, by Theorem 2 we deduce that $V^{\prime} \subseteq W_{0}$ and $\Gamma^{\prime}$ induces a winning strategy for player 0 on $\Gamma$.

Theorem 6. Given an energy game $\Gamma=\left(V, E, v_{0}, w,\left\langle V_{0}, V_{1}\right\rangle\right)$, the size of the propositional logic formula $\phi_{\Gamma}^{\prime}$ is $\mathcal{O}(|E| \cdot\lceil\log ((V+1) \cdot W)\rceil)$, even if $\phi_{\Gamma}^{\prime}$ is required to be in $C N F$.

\section{CONCLUSIONS}

We devise efficient encodings of the energy games problem into the satisfiability problem for formulas of difference logic and pure propositional logic in conjunctive normal form. Tight upper bounds on the sizes of the given reductions are also reported. Due to the success of nowadays SAT solvers in symbolic verification, the proposed encodings could result in algorithms that are very efficient in practice. Furthermore, they could open up new possibilities for devising tight bounds on the complexity of the energy games problem, as there are fragments of SAT that can be solved in polynomial time. 


\section{REFERENCES}

[1] A. Ehrenfeucht and J. Mycielski. International journal of game theory. Positional Strategies for Mean-Payoff Games, 8:109-113, 1979.

[2] P. Bouyer, U. Fahrenberg, K. G. Larsen, N. Markey, and J. Srba. Infinite runs in weighted timed automata with energy constraints. In Proc. of FORMATS: Formal Modeling and Analysis of Timed Systems, LNCS 5215, pages 33-47. Springer, 2008.

[3] A. Chakrabarti, L. de Alfaro, T. A. Henzinger, and M. Stoelinga. Resource interfaces. In Proc. of EMSOFT: Embedded Software, LNCS 2855, pages 117-133. Springer, 2003.

[4] Y. Gurevich and L. Harrington. Trees, automata, and games. In Proc. of STOC: Symposium on Theory of Computing, pages 60-65. ACM, 1982.

[5] Keijo Heljanko, Misa Kein“anen, Martin Lange, and Ilkka Niemel“a. Solving parity games by a reduction to sat. J. Comput. Syst. Sci., 78(2):430-440, March 2012.

[6] M. Jurdzinski. Small progress measures for solving parity games. In Proceedings of STACS: Theoretical Aspects of Computer Science, LNCS 1770, pages 290-301. Springer, 2000.

[7] L. Brim, J. Chaloupka, L. Doyen, R. Gentilini, and J-F. Raskin. Faster algorithms for mean payoff games. Formal Methods in System Design, 38(2):97-118, 2011.

[8] Moez Mahfoudh, Peter Niebert, Eugene Asarin, and Oded Maler. A satisfiability checker for difference logic. In 5-th Int. Symp. on the Theory and Applications of Satisfliability Testing, 2002. 